\title{
Correction to: Prediction of femoral strength using 3D finite element models reconstructed from DXA images: validation against experiments
}

\author{
Lorenzo Grassi $^{1}$ (1) · Sami P. Väänänen ${ }^{2,3} \cdot$ Matti Ristinmaa ${ }^{4}$ Jukka S. Jurvelin ${ }^{2,5} \cdot$ Hanna Isaksson ${ }^{1}$
}

Published online: 27 May 2019

(c) Springer-Verlag GmbH Germany, part of Springer Nature 2019

\section{Correction to: \\ Biomech Model Mechanobiol (2017) 16:989-1000 https://doi.org/10.1007/s10237-016-0866-2}

In the original publication of the article, Fig. 3 and Tables 2 , 4 and 5 were published with errors. The issue was caused by an error in the code used to predict femoral strength in the finite element (FE) models, and that was presented in a previous publication (Grassi et al. 2016). For any details about the error and how that was addressed, we refer the interested reader to the corrigendum that was published for that publication (Grassi et al. 2019).

The correct Fig. 3 and Tables 2, 4 and 5 are provided below. The overall accuracy of the new results was similar to that in the original article. Thus, the overall findings and conclusions from the original article are still valid.
The original article can be found online at https://doi.org/10.1007/ s10237-016-0866-2.

Lorenzo Grassi

lorenzo.grassi@bme.lth.se

1 Department of Biomedical Engineering, Lund University, BMC D13, 22184 Lund, Sweden

2 Department of Applied Physics, University of Eastern Finland, Kuopio, Finland

3 Department of Orthopaedics, Traumatology and Hand Surgery, Kuopio University Hospital, Kuopio, Finland

4 Division of Solid Mechanics, Lund University, Lund, Sweden

5 Diagnostic Imaging Center, Kuopio University Hospital, Kuopio, Finland 

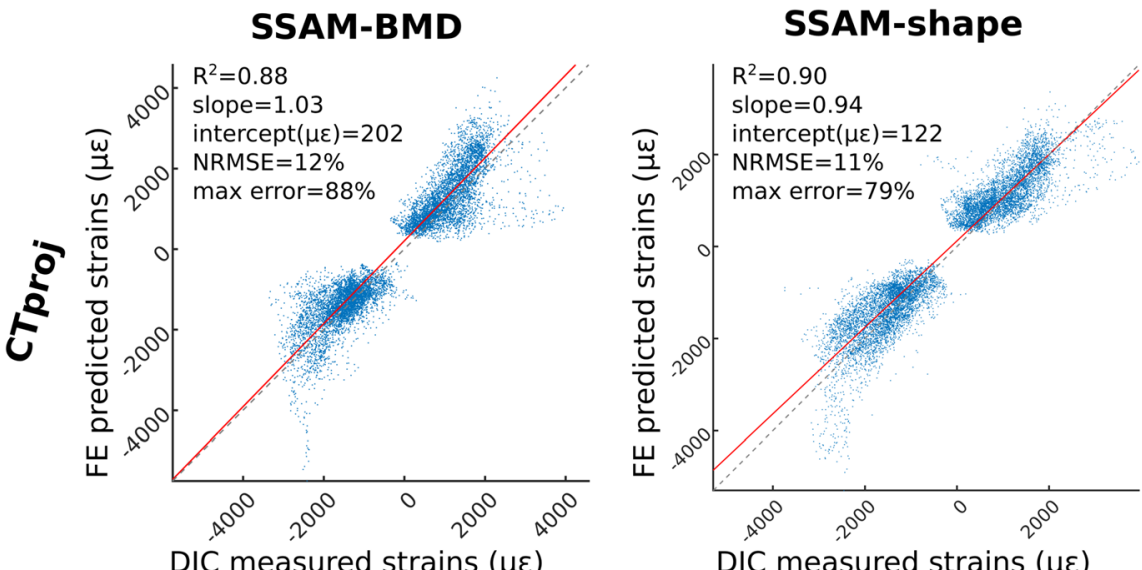

DIC measured strains $(\mu \varepsilon)$

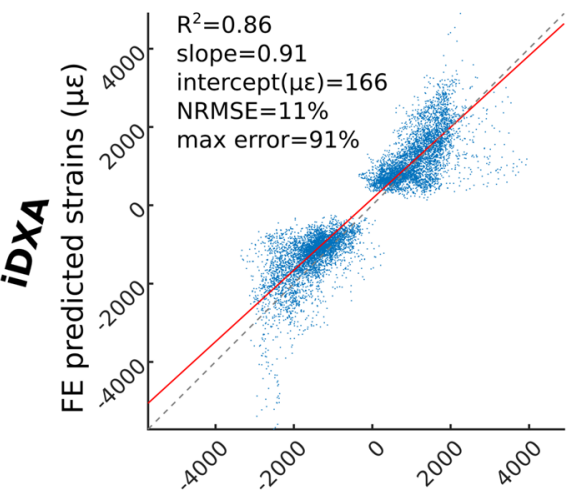

DIC measured strains $(\mu \varepsilon)$

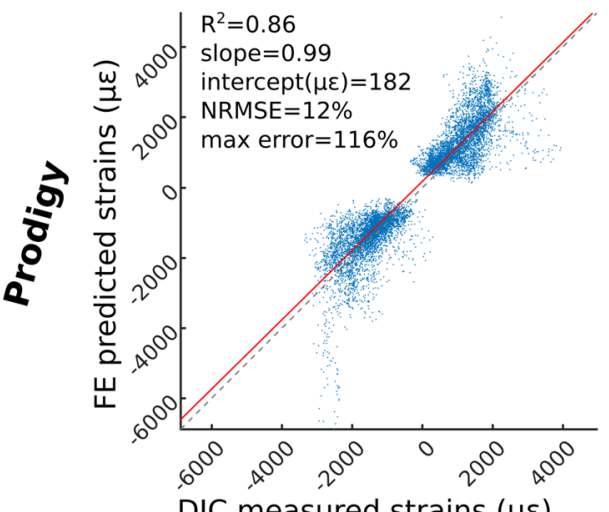

DIC measured strains $(\mu \varepsilon)$

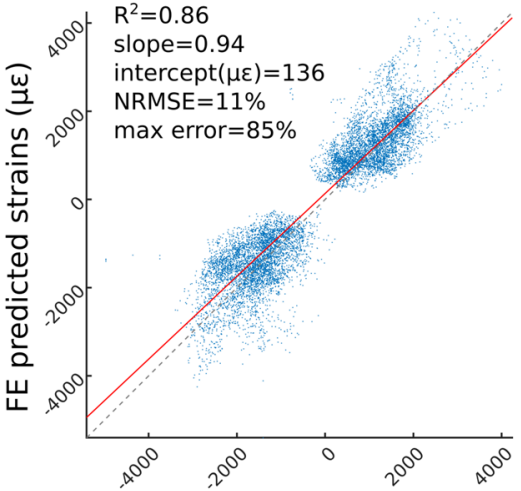

DIC measured strains $(\mu \varepsilon)$

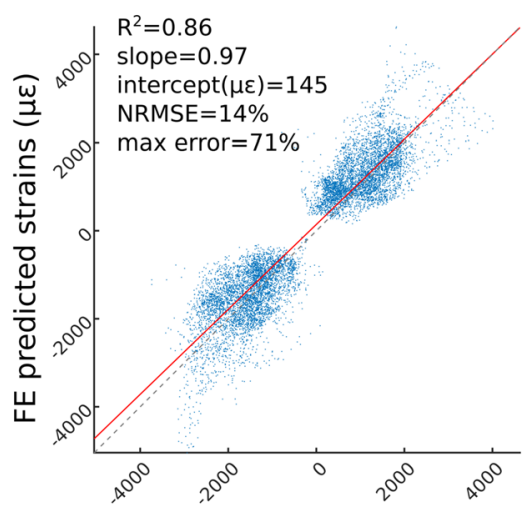

DIC measured strains $(\mu \varepsilon)$
SSAM-shape\&BMD

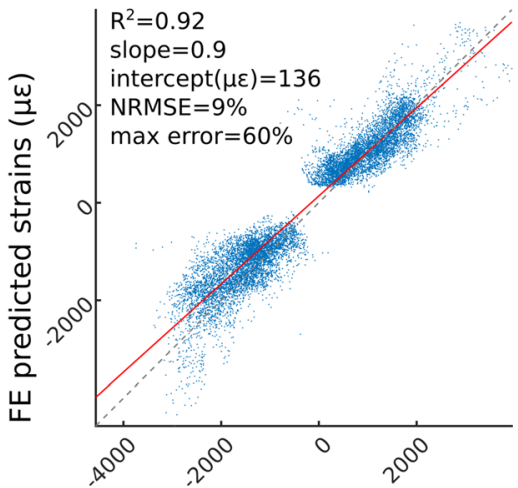

DIC measured strains $(\mu \varepsilon)$

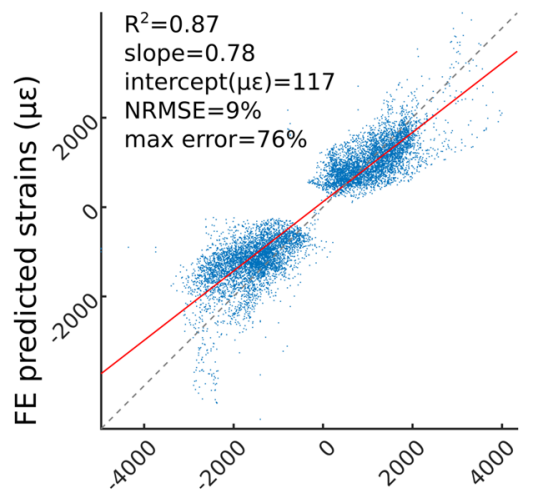

DIC measured strains $(\mu \varepsilon)$

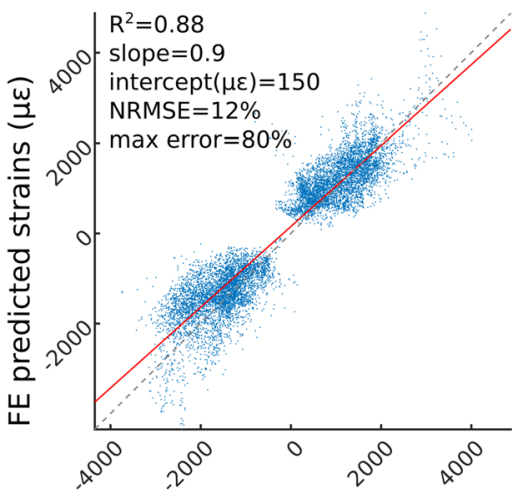

DIC measured strains $(\mu \varepsilon)$
Fig. 3 Prediction accuracy for the major and minor principal strains for SSAM-BMD (first column), SSAM-shape (second column), and SSAM-shape and BMD (third column) models for the three bones pooled together. From top to bottom, the accuracy results are plotted for the models using CT projection, iDXA, and Prodigy images for the SSAM-based reconstructions. Only the middle column (SSAMshape) is affected by the corrigendum 
Table 2 Prediction accuracy for the major and minor principal strains for SSAMbased models of the three bones taken individually. Only the second row (SSAM-shape) and the reference data from CT-based models (Grassi et al. 2019, 2016) are affected by the corrigendum

\begin{tabular}{|c|c|c|c|c|c|c|c|c|c|}
\hline & \multicolumn{3}{|l|}{ Bone \#1 } & \multicolumn{3}{|l|}{ Bone \#2 } & \multicolumn{3}{|c|}{ Bone \#3 } \\
\hline & CTproj & iDXA & Prodigy & CTproj & iDXA & Prodigy & CTproj & iDXA & Prodigy \\
\hline \multicolumn{10}{|l|}{ SSAM-BMD } \\
\hline $\mathrm{R}^{2}$ & 0.9 & 0.9 & 0.9 & 0.84 & 0.83 & 0.83 & 0.92 & 0.89 & 0.89 \\
\hline Slope & 1 & 0.91 & 0.92 & 1.03 & 0.99 & 1.08 & 1.03 & 0.85 & 0.97 \\
\hline Intercept $(\mu \varepsilon)$ & 225 & 199 & 200 & 257 & 263 & 283 & 142 & 84 & 107 \\
\hline NRMSE (\%) & 13 & 11 & 11 & 19 & 18 & 20 & 12 & 12 & 12 \\
\hline Max error (\%) & 64 & 69 & 70 & 89 & 89 & 113 & 63 & 58 & 80 \\
\hline \multicolumn{10}{|l|}{ SSAM-shape } \\
\hline $\mathrm{R}^{2}$ & 0.90 & 0.85 & 0.84 & 0.91 & 0.91 & 0.89 & 0.91 & 0.87 & 0.89 \\
\hline Slope & 0.87 & 0.93 & 1.01 & 0.91 & 0.82 & 0.84 & 1.07 & 1.17 & 1.16 \\
\hline Intercept $(\mu \varepsilon)$ & 195 & 263 & 299 & 65 & 100 & 94 & 54 & 76 & 91 \\
\hline NRMSE (\%) & 12 & 15 & 17 & 12 & 12 & 13 & 10 & 11 & 14 \\
\hline Max error (\%) & 37 & 46 & 82 & 79 & 49 & 66 & 82 & 88 & 84 \\
\hline \multicolumn{10}{|c|}{ SSAM-shape and BMD } \\
\hline $\mathrm{R}^{2}$ & 0.88 & 0.84 & 0.86 & 0.94 & 0.89 & 0.9 & 0.92 & 0.87 & 0.88 \\
\hline Slope & 0.81 & 0.76 & 0.88 & 0.9 & 0.74 & 0.86 & 0.98 & 0.86 & 0.99 \\
\hline Intercept $(\mu \varepsilon)$ & 197 & 217 & 252 & 109 & 141 & 181 & 68 & 3 & 17 \\
\hline NRMSE $(\%)$ & 11 & 14 & 15 & 10 & 11 & 13 & 9 & 8 & 12 \\
\hline Max error $(\%)$ & 34 & 37 & 43 & 51 & 70 & 61 & 74 & 72 & 91 \\
\hline \multicolumn{10}{|c|}{ CT-based (Grassi et al. 2016) } \\
\hline $\mathrm{R}^{2}$ & 0.92 & & & 0.93 & & & 0.94 & & \\
\hline Slope & 0.93 & & & 0.92 & & & 1.00 & & \\
\hline Intercept $(\mu \varepsilon)$ & 148 & & & 111 & & & 64 & & \\
\hline NRMSE (\%) & 11 & & & 11 & & & 10 & & \\
\hline Max error $(\%)$ & 41 & & & 52 & & & 59 & & \\
\hline
\end{tabular}

For each bone, the accuracy obtained using the three different 2D reference images (CT projection, iDXA, and Prodigy) for the SSAM-based reconstruction is reported. The accuracy parameters reported by Grassi et al. (2016) for the CT-based models are also reported in the last row to allow for an easy comparison 
Table 4 Prediction accuracy for the major and minor principal strains in the femoral neck region only for SSAM-based models, for the three bones pooled and for each individual bone. Only the second row
(SSAM-shape) and the reference data from CT-based models (Grassi et al. 2019, 2016) are affected by the corrigendum

\begin{tabular}{|c|c|c|c|c|c|c|c|c|c|c|c|c|}
\hline & \multicolumn{3}{|c|}{3 bones pooled } & \multicolumn{3}{|l|}{ Bone \#1 } & \multicolumn{3}{|l|}{ Bone \#2 } & \multicolumn{3}{|c|}{ Bone \#3 } \\
\hline & CTproj & iDXA & Prodigy & CTproj & iDXA & Prodigy & CTproj & iDXA & Prodigy & CTproj & iDXA & Prodigy \\
\hline \multicolumn{13}{|l|}{ SSAM-BMD } \\
\hline $\mathrm{R}^{2}$ & 0.81 & 0.77 & 0.76 & 0.83 & 0.83 & 0.83 & 0.76 & 0.73 & 0.74 & 0.88 & 0.83 & 0.81 \\
\hline Slope & 0.84 & 0.76 & 0.83 & 0.90 & 0.87 & 0.84 & 0.77 & 0.77 & 0.91 & 0.86 & 0.69 & 0.80 \\
\hline Intercept $(\mu \varepsilon)$ & 103 & 80 & 94 & 245 & 249 & 235 & -39 & -42 & -56 & 37 & -19 & -11 \\
\hline NRMSE (\%) & 10 & 11 & 9 & 17 & 16 & 15 & 22 & 25 & 29 & 14 & 14 & 17 \\
\hline Max error (\%) & 67 & 68 & 71 & 72 & 74 & 77 & 98 & 95 & 115 & 70 & 66 & 88 \\
\hline \multicolumn{13}{|l|}{ SSAM-shape } \\
\hline $\mathrm{R}^{2}$ & 0.87 & 0.86 & 0.85 & 0.90 & 0.86 & 0.84 & 0.87 & 0.91 & 0.89 & 0.88 & 0.85 & 0.88 \\
\hline Slope & 0.91 & 0.97 & 1.00 & 0.87 & 0.90 & 0.95 & 0.91 & 0.88 & 0.90 & 0.98 & 1.27 & 1.27 \\
\hline Intercept $(\mu \varepsilon)$ & 68 & 122 & 161 & 190 & 270 & 342 & -97 & -33 & -16 & 28 & 215 & 218 \\
\hline NRMSE (\%) & 10 & 15 & 13 & 11 & 15 & 17 & 19 & 14 & 16 & 11 & 12 & 16 \\
\hline Max error $(\%)$ & 59 & 78 & 57 & 38 & 46 & 84 & 75 & 42 & 59 & 73 & 95 & 91 \\
\hline \multicolumn{13}{|c|}{ SSAM-shape and BMD } \\
\hline $\mathrm{R}^{2}$ & 0.89 & 0.85 & 0.86 & 0.88 & 0.83 & 0.83 & 0.93 & 0.87 & 0.89 & 0.88 & 0.84 & 0.86 \\
\hline Slope & 0.89 & 0.78 & 0.91 & 0.79 & 0.71 & 0.81 & 0.95 & 0.75 & 0.88 & 0.95 & 0.93 & 1.08 \\
\hline Intercept $(\mu \varepsilon)$ & 163 & 110 & 154 & 269 & 245 & 291 & 27 & 27 & 67 & 60 & 51 & 84 \\
\hline NRMSE (\%) & 10 & 12 & 12 & 12 & 14 & 16 & 13 & 14 & 16 & 11 & 10 & 14 \\
\hline Max error (\%) & 51 & 65 & 66 & 41 & 35 & 39 & 45 & 66 & 57 & 71 & 74 & 91 \\
\hline \multicolumn{13}{|c|}{ CT-based (Grassi et al. 2016) } \\
\hline $\mathrm{R}^{2}$ & 0.90 & & & 0.88 & & & 0.90 & & & 0.93 & & \\
\hline Slope & 0.86 & & & 0.90 & & & 0.83 & & & 0.88 & & \\
\hline Intercept $(\mu \varepsilon)$ & 77 & & & 192 & & & -22 & & & 37 & & \\
\hline NRMSE (\%) & 11 & & & 14 & & & 14 & & & 10 & & \\
\hline Max error $(\%)$ & 46 & & & 40 & & & 49 & & & 44 & & \\
\hline
\end{tabular}

The accuracy obtained using the three different 2D reference images (CT projection, iDXA, and Prodigy) for the SSAM-based reconstruction is reported. The accuracy parameters reported by the CT-based FE models presented in Grassi et al. (2016) for the femoral neck region only are also reported in the last row to allow for a direct comparison

Table 5 Femoral strength prediction accuracy for bones \#1 and \#2, for the three different FE models (SSAM-BMD, SSAM-shape, and SSAM-shape and BMD), each of them built for the three different 2D reference images (CT projection, iDXA, and Prodigy). All computational data in table is affected by the corrigendum

\begin{tabular}{|c|c|c|c|c|c|c|c|}
\hline & \multicolumn{3}{|l|}{ Bone \#1 } & \multicolumn{3}{|l|}{ Bone \#2 } & \multirow[t]{2}{*}{ SEE $[N]$} \\
\hline & CTproj & iDXA & Prodigy & CTproj & iDXA & Prodigy & \\
\hline SSAM-BMD & $10,821(-19 \%)$ & $11,043(-17 \%)$ & $11,242(-16 \%)$ & $7155(-9 \%)$ & $5945(-24 \%)$ & $5593(-29 \%)$ & 2077 \\
\hline SSAM-shape & $13,158(-2 \%)$ & $12,373(-8 \%)$ & $10,900(-19 \%)$ & $8229(+5 \%)$ & $8724(+11 \%)$ & $7879(+0 \%)$ & 1164 \\
\hline SSAM-shape and BMD & $14,946(+12 \%)$ & $13,427(+0 \%)$ & $12,070(-10 \%)$ & $7516(-4 \%)$ & $8873(+13 \%)$ & $7548(-4 \%)$ & 950 \\
\hline CT-based (Grassi et al. 2016) & $13,144(-1.8 \%)$ & & & $7663(-2.5 \%)$ & & & 403 \\
\hline $\begin{array}{l}\text { Experimentally measured } \\
\text { (Grassi et al. 2014) }\end{array}$ & 13,383 & & & 7856 & & & - \\
\hline
\end{tabular}

The relative error to the actual femoral strength measured experimentally (Grassi et al. 2014) is reported between parentheses. The strength prediction accuracy reported by Grassi et al. (2016) for the CT-based models is also reported in the last row to allow for an easy comparison 


\section{Reference}

Grassi L, Väänänen SP, Amin Yavari S, Jurvelin JS, Weinans H, Ristinmaa M, Zadpoor AA, Isaksson H (2014) Full-field strain measurement during mechanical testing of the human femur at physiologically relevant strain rates. J Biomech Eng 136:111010. https://doi.org/10.1115/1.4028415

Grassi L, Väänänen SP, Ristinmaa M, Jurvelin JS, Isaksson H (2016) How accurately can subject-specific finite element models predict strains and strength of human femora? Investigation using full-field measurements. J Biomech 49:802-806. https://doi. org/10.1016/j.jbiomech.2016.02.032
Grassi L, Väänänen SP, Ristinmaa M, Jurvelin JS, Isaksson H (2019) Corrigendum to "How accurately can subject-specific finite element models predict strains and strength of human femora?" Investigation using full-field measurements. J Biomech 49:802806. https://doi.org/10.1016/j.jbiomech.2018.12.032

Publisher's Note Springer Nature remains neutral with regard to jurisdictional claims in published maps and institutional affiliations. 